TITLE:

\title{
An adaptive supramolecular hydrogel comprising self-sorting double nanofibre networks
}

\section{$\operatorname{AUTHOR}(\mathrm{S})$ :}

Shigemitsu, Hajime; Fujisaku, Takahiro; Tanaka, Wataru; Kubota, Ryou; Minami, Saori; Urayama, Kenji; Hamachi, Itaru

\section{CITATION:}

Shigemitsu, Hajime ...[et al]. An adaptive supramolecular hydrogel comprising self-sorting double nanofibre networks. Nature Nanotechnology 2018, 13(2): 165-172

\section{ISSUE DATE:}

2018-01-08

URL:

http://hdl.handle.net/2433/231032

\section{RIGHT:}

This is a post-peer-review, pre-copyedit version of an article published in 'Nature Nanotechnology'. The final authenticated version is available online at: https://doi.org/10.1038/s41565-017-0026-6.; The full-text file will be made open to the public on 8 July 2018 in accordance with publisher's 'Terms and Conditions for Self-Archiving'; この論文は 出版社版でありません。引用の際には出版社版をご確認ご利用ください。; This is not the published version. Please cite only the published version. 
1 An adaptive supramolecular hydrogel comprising a self-sorting double nanofibre

2 network

3

4 Hajime Shigemitsu ${ }^{1, \dagger}$, Takahiro Fujisaku ${ }^{1}$, Wataru Tanaka ${ }^{1}$, Ryou Kubota ${ }^{1}$, Saori Minami ${ }^{2}$,

$5 \quad$ Kenji Urayama ${ }^{2}$, Itaru Hamachi ${ }^{1,3 *}$

6

$7 \quad{ }^{1}$ Department of Synthetic Chemistry and Biological Chemistry, Graduate School of

8 Engineering, Kyoto University, Katsura, Kyoto 615-8510, JAPAN

$9{ }^{2}$ Department of Macromolecular Science and Engineering, Kyoto Institute of Technology,

10 Matsugasaki, Kyoto 606-8585, JAPAN

$11{ }^{3}$ Core Research for Evolutional Science and Technology (CREST), Japan Science and

12 Technology Agency (JST), 5 Sanbancho, Chiyoda-ku, Tokyo 102-0075, JAPAN

13 'Present address: Department of Applied Chemistry, Graduate School of Engineering, Osaka

14 University, 2-1 Yamadaoka, Suita, Osaka 565-0871, JAPAN

15

16

17 Correspondence: ihamachi@sbchem.kyoto-u.ac.jp

18 


\section{Abstract}

2 Novel soft materials should comprise multiple supramolecular nanostructures whose 3 responses (e.g. assembly and disassembly) to external stimuli can be controlled 4 independently. Such multicomponent systems are present in living cells and control the

5 formation and breaking up of a variety of supramolecular assemblies made of proteins, lipids,

6 DNA and RNA in response to external stimuli; however, artificial counterparts are

7 challenging to make. Here, we present a hybrid hydrogel consisting of a self-sorting double

8 network (SDN) of nanofibres in which each network responds to an applied external stimulus

9 independent of the other. The hydrogel can be made to change its mechanical properties and

10 rates of release of encapsulated proteins by adding $\mathrm{Na}_{2} \mathrm{~S}_{2} \mathrm{O}_{4}$ or bacterial alkaline phosphatase

11 (BAP). Notably, the properties of the gel depend on the order in which the external stimuli

12 are applied. Multicomponent hydrogels comprising orthogonal stimulus-responsive

13 supramolecular assemblies would be suitable for designing novel adaptive materials. 


\section{$1 \quad$ Main text}

2 In living systems, a myriad of biomolecules such as proteins, lipids, DNA, and RNA

3 spontaneously form distinct supramolecular assemblies through noncovalent interactions. ${ }^{1}$

4 These assemblies are diverse and orthogonal and their formation and collapse are precisely

5 regulated, which is crucial for the multifunctionality and autonomy of living cells. Fibrous

6 supramolecular assemblies of cells (e.g., actin filaments, and microtubules) respond to

7 various chemical and physical stimuli (e.g., $\mathrm{pH}$, mechanical stress, and biomolecules), and

8 dynamic structural changes are closely involved in the mechanical support of live cells, their

9 metamorphosis, and migration. ${ }^{2}$ Given such sophisticated natural systems, the controlled

10 hybridisation of multiple supramolecular assemblies bearing orthogonal functionality is

11 expected to produce novel artificial soft materials. ${ }^{3-19}$ Self-sorting events and orthogonal

12 assemblies of synthetic molecules are among the key factors for the construction of ordered

13 systems made of multiple supramolecular structures. van Esch and co-workers pioneered the

14 demonstration of hybrid supramolecular hydrogels by orthogonally encapsulating vesicles

15 that contained enzymes. ${ }^{20}$ Adams and co-workers prepared a self-sorting double network

16 (SDN) by applying gradually changing the $\mathrm{pH}^{21}$ They subsequently developed SDN

17 hydrogels that can be spatially resolved using UV light. ${ }^{22}$ Smith and co-workers also reported

18 a phototuneable SDN hydrogel containing a photo acid generator. ${ }^{23}$ Additionally,

19 catalytically incompatible groups can be incorporated into self-sorting nanofibres to achieve

20 multi-step reactions that would not be possible in solution. ${ }^{24}$ Self-sorting n-type and p-type

21 supramolecular nanofibres can be assembled for new electrical properties. ${ }^{25,26}$ The

22 preparation of supramolecular assemblies/polymer hybrid materials has also been reported. ${ }^{27}$

23 However, the development of hybrid systems composed of supramolecules responsive to 
1 multiple (and orthogonal) stimuli still remains challenging. Such adaptive supramolecular

2 materials exhibiting multiple and unique responses to chemical/biological stimuli could open

3 up the next generation of smart materials for a variety of applications. Their design strategy

4 has not yet well been explored, unlike materials that rely on co-assembly.

Herein, we discovered a new self-sorting gelator pair and a supramolecular

6 hydrogel comprising a stimulus-responsive double network (Figure 1a). Two supramolecular

7 nanofibres that form the SDN hydrogel orthogonally responded orthogonally to different

8 stimuli $\left(\mathrm{Na}_{2} \mathrm{~S}_{2} \mathrm{O}_{4}\right.$ and bacterial alkaline phosphatase (BAP)) and the SDN hydrogel showed

9 bidirectional change in the macroscopic rheological properties. Due to the self-sorting of the

10 two nanofibres, each property could be imparted without interference upon mixing.

11 Unprecedented bidirectional rheological changes led to a unique application in the

12 bidirectionally tuneable release of proteins encapsulated in the SDN hydrogel. Furthermore,

13 the SDN hydrogels exhibited the unique adaptive feature of stimulus-order recognition,

14 which was expressed by the macroscopic gel-sol transition. 


\section{Exploration and evaluation of self-sorting double nanofibre networks}

2 We recently reported that two distinct supramolecular fibres $-\mathbf{B P m o c}_{\mathbf{3}}{ }^{28}$ and

3 Phos-cycC $_{6}{ }^{29}$ - are self-sorting (Figure 1b, Supplementary Figure 1), which could be clearly

4 visualised in situ using confocal laser scanning microscopy (CLSM). ${ }^{30}$ Our previous study

5 suggested that orthogonal interaction modes between two components may be one of the

6 strategies for self-sorting; various factors such as formation (kinetic) processes are significant

7 in this event. ${ }^{14,21}$ In our cases, the large difference in the interaction modes between

8 peptide-type $\mathbf{B P m o c}-\mathbf{F}_{3}$ (mainly governed by $\pi-\pi$ interactions and hydrogen bonding) and

9 lipid-type Phos-cycC $_{6}$ (through van der Waals interactions and hydrogen bonding) showed

10 preference for self-sorting rather than co-assembly. ${ }^{31}$ On the basis of this assumption, we

11 sought to explore a new self-sorting supramolecular fibre pair based on the combination of

12 peptide-type and lipid-type hydrogelators, and found that NPmoc-FF derivatives ${ }^{32,33}$ — in

13 which the N-terminal group is replaced with a nitrobenzyl from the boronobenzyl of

14 BPmoc-F $\mathbf{F}_{3}$ - can be self-sorted with Phos-cycC $\mathbf{C}_{6}$ (Figure 1b). Orthogonality in the

15 two-component hydrogel comprising NPmoc-FF or NPmoc-F(4-F)F and Phos-cycC $_{6}$ was

16 initially confirmed by circular dichroism (CD) spectroscopy. NPmoc-F(4-F)F and

17 Phos-cycC $_{6}$ in a single-component system display a different pattern in the

18 assembly-enhanced CD spectra in an aqueous buffer $(100 \mathrm{mM}$ HEPES

19 (4-(2-hydroxyethyl)-1-piperazineethanesulfonic acid), $\mathrm{pH} \quad 8.0$ ) (Figure 2a, b).

20 NPmoc-F(4-F)F, in particular, showed characteristic negative Cotton peaks (273 nm), which

21 were derived from the self-assembled $\beta$-sheet-like structure (Figure 2a, Supplementary

22 Figure 2). An assembly-enhanced broad peak was also detected at approximately $265 \mathrm{~nm}$

23 (negative Cotton effect) in a viscous solution of Phos-cycC $\mathbf{~}_{\mathbf{6}}$ (Figure 2b, Supplementary 
1 Figure 2), which suggested that assemblies were formed even below the critical gelation

2 concentration (CGC). The $\mathrm{CD}$ spectrum of a mixture of two components generating 3 self-sorting supramolecular nanofibres should be a simple sum of each component 4 (theoretical CD spectrum). As expected, the experimental CD agreed with the theoretical CD 5 spectrum (Figure 2c). The self-sorting structures were retained throughout the use of varied

6 mixture ratios or concentrations (Supplementary Figure 3). Similarly, the experimental CD

7 spectrum of the NPmoc-FF and Phos-cycC $\mathbf{C}_{6}$ mixture corresponded to the sum of the two 8 corresponding CD spectra (Supplementary Figure 4).

9

Self-sorting of nanofibres in the hydrogels was further confirmed by in situ imaging using CLSM. ${ }^{30,34}$ To selectively stain NPmoc-FF or NPmoc-F(4-F)F fibres, we designed and synthesised a fluorescent probe (NP-Alexa647) bearing the NPmoc-FF moiety as the hydrophobic core and anionic Alexa647 dye in the hydrophilic part (Figure 1b). To image Phos-cycC 6 fibres, NBD-cycC 6 comtaining a core similar to Phos-cycC $_{6}$ was employed (Figure 1b). We then checked the staining selectivities of the two probes for the corresponding fibres. As shown in Figure 2d and e, NP-Alexa647 stained NPmoc-F(4-F)F fibres but not Phos-cycC $_{6}$ fibres, whereas NBD-cycC $_{6}$ stained Phos-cycC $_{6}$ fibres but not NPmoc-F(4-F)F fibres. With these selective probes, we subsequently sought to visualise the two self-sorting fibres containing NPmoc-F(4-F)F and Phos-cycC $_{6}$ in the mixed hydrogel (Supplementary Figure 8). The four components, - NPmoc-F(4-F)F, NP-Alexa647, Phos-cycC $_{6}$, and NBD-cycC $_{6}$ - were mixed and the suspension was heated to form a homogeneous solution, followed by cooling to room temperature to form the gel. The resultant hydrogel was observed using CLSM. Images through the channels for Alexa647 and 
1 NBD showed fibrous structures, but their spatial distributions were visibly different. We

2 quantitatively evaluated the self-sorting degree via Pearson's correlation coefficient $(r)$ which

3 is used to measure the strength of a linear association between two variables. ${ }^{35}$ The Pearson's

4 correlation coefficients of the merged images were in the range $0.20-0.30$, indicating

5 negligible correlation between the two original images (Supplementary Figure 8). In addition,

6 the super-resolution CLSM images with an Airyscan unit (Figure 2f, Supplementary Figure

7 9) clearly showed distinct fibres in the SDN hydrogel (Pearson's correlation coefficients: -

8 0.25-0.30). The data showed that almost all NPmoc-F(4-F)F and Phos-cycC 6 were

9 self-sorted and orthogonally assembled into two distinct supramolecular nanofibres, thereby

10 forming an SDN hydrogel. Fibers of NPmoc-FF were too thin to be observed clearly, as

11 shown in Supplementary Figure 10, moreover, the CLSM image of the mixture

12 (NPmoc-FF/Phos-cycC 6 /NP-Alexa647/NBD-cycC ( $_{6}$ ) also did not clearly show self-sorting

13 structures (Supplementary Figure 10). Therefore, we could not clearly observe the

14 self-sorting of NPmoc-FF and Phos-cycC $_{6}$, and decided to use NPmoc-F(4-F)F 
1 Stimulus-response behaviours of a single-component NPmoc-F(4-F)F and Phos-cycC 6

2

\section{networks}

Prior to exploring the functions of the hydrogel containing the two-component self-sorting supramolecular nanofibres, we evaluated the response properties of each single component, i.e. the NPmoc-F(4-F)F hydrogel and $\mathbf{P h o s}_{-} \mathbf{c y c C}_{\mathbf{6}}$ viscous liquid. As reported previously, ${ }^{32}$ the NPmoc moiety was reductively eliminated, causing the gel-sol transition of the NPmoc-F(4-F)F hydrogel in response to appropriate reducing reagents such as $\mathrm{Na}_{2} \mathrm{~S}_{2} \mathrm{O}_{4}$ (Figure 3a). As shown in Figure 3b, the NPmoc-F(4-F)F hydrogel rapidly changed to sol upon addition of $\mathrm{Na}_{2} \mathrm{~S}_{2} \mathrm{O}_{4}$. The HPLC analysis showed that $>99 \%$ NPmoc-F(4-F)F was decomposed by $\mathrm{Na}_{2} \mathrm{~S}_{2} \mathrm{O}_{4}$ within 10 min (Figure 3c, Supplementary Figure 11); the CD spectrum of the resultant sol did not show a Cotton peak at approximately $273 \mathrm{~nm}$, which was originally detected as a result of the $\beta$-sheet-like structure of the NPmoc-F(4-F)F nanofibres (Figure 3d). It was clear that NPmoc-F(4-F)F nanofibres were destroyed by the chemical reaction with $\mathrm{Na}_{2} \mathrm{~S}_{2} \mathrm{O}_{4}$, similar to the case of the NPmoc-FF fibres.

We also explored the stimulus-responsiveness of a single-component Phos-cyc $_{6}$ fibres by treating them with bacterial alkaline phosphatase (BAP), an enzyme that hydrolyses organophosphates (Figure 3e). BAP had a clear effect on Phos-cycC $\mathbf{C}_{\mathbf{6}}$ fibres, as demonstrated by the gradual change of the viscous solution of Phos-cycC $\mathbf{C}_{6}(0.15 \mathrm{wt} \%$ : below the CGC $(0.30 \mathrm{wt} \%))$ to a transparent gel $2 \mathrm{~h}$ after the addition of BAP (Figure $3 \mathrm{f}$ ). The macroscopic sol-gel change observed by the naked eye was consistent with the rheological measurements (Figure 3g, Supplementary Figure 14). The $G^{\prime}$ value of the Phos-cycC 6 viscous liquid was already larger than the $G^{\prime \prime}$ value before BAP addition, indicating the existence of loosely linked fibres suggested by the CD spectra (Figure $2 b$ ). The addition of BAP continuously 
1 increased the $G^{\prime}$ value for $3 \mathrm{~h}$, whereas the $G^{\prime \prime}$ value remained almost constant, resulting in a

2 greater $G^{\prime}(244 \mathrm{~Pa})$ relative to $G^{\prime \prime}(16 \mathrm{~Pa})(\tan \delta$ : 0.07$)$. Gelation was attributed to the

3 BAP-catalysed hydrolysis of Phos-cycC $_{6}$ to produce hydrophobic $\mathbf{H O}-\mathbf{c y c C}_{6}$ (Figure $3 \mathrm{e}$ )

4 which is insoluble in the aqueous buffer solution. HPLC product analysis indicated partial

5 conversion of Phos-cyc $_{6}$ to $\mathbf{H O}-\mathbf{c y c C}_{6}$ (Figure 3h, Supplementary Figure 12), whereby the

6 Phos-cycC $\mathbf{C}_{6}$ fraction gradually decreased with the concurrent increase of HO-cycC $_{6}$.

7 Interestingly, gelation took place when approximately $50 \%$ of $\mathbf{P h o s}-\mathbf{c y c C}_{6}$ was hydrolysed

8 (Supplementary Figure 13).

To examine the detailed mechanism of the BAP-induced gelation of Phos-cycC $_{6}$, we carefully observed changes to the Phos-cycC $_{6}$ fibre network using CLSM in situ

11 (Supplementary Figure 15). Many fibres were observed even in the viscous liquid state

12 (before BAP addition), which was in agreement with the data from the rheological

13 experiments and CD spectroscopy. The morphology and density of these fibres did not

14 changed substantially after the addition of BAP. However, we found that the fibre fluidity,

15 which was monitored by fluorescent recovery after photobleaching (FRAP) technique, ${ }^{36,37,38}$

16 was greatly altered by the BAP treatment (Supplementary Figure 16). Before the addition of

17 BAP, the bleached fluorescence recovered sufficiently (recovery ratio: $92.1 \pm 4.7 \%$ ) within

183 min, indicating that the $\mathbf{P h o s}_{-\mathbf{c y c C}} \mathbf{6}$ fibres were fluidic (diffusion coefficient $(D): 1.9 \pm$

$\left.190.4 \times 10^{-3} \mu \mathrm{m}^{2} / \mathrm{sec}\right)$. In contrast, the fibres treated with BAP showed negligible

20 fluorescence recovery (recovery ratio: $3.3 \pm 3.3 \%$ ), suggesting the presence of less fluidic

21 solid-like fibres. This remarkable difference may be ascribed to surface modification and/or rearrangement of intermolecular hydrogen bonding of the supramolecular fibres by BAP.

Additional CD experiments also showed the impact of BAP treatment on the fibres. The 
1 assembly-enhanced CD spectrum was observed before the BAP addition and it remained

2 almost intact with a slight shift of the Cotton peak from 265 to $272 \mathrm{~nm}$ (Figure 3i). This

3 strongly suggested that the molecular packing/orientation of the original Phos-cycC $_{6}$ fibre

4 was modulated by the BAP-catalysed partial hydrolysis. Detailed experiments

5 (Supplementary Figures 17-20) suggested that 'enzymatic modification of supramolecular

6 assemblies' may be plausible in this case, where the partial conversion of $\mathbf{P h o s}^{-} \mathbf{c y c}_{\mathbf{6}} \mathbf{C}_{\mathbf{6}}$ to

7 HO-cycC $\mathbf{C}_{6}$ by BAP occurred at the supramolecular fibres surfaces and/or ends.

To investigate changes in the gel network more precisely, we used nanobeads with

9 various diameters $(70-800 \mathrm{~nm})$ embedded in the network. The movement of nanobeads was

10 traced using CLSM, which allowed us to evaluate the size of meshes formed by entangled

11 supramolecular fibres. ${ }^{39,40}$ When the mesh size of the fibrous network was smaller than that

12 of fluorescent beads, Brownian motion of the beads was restricted and stopped, whereas the

13 beads moved freely when the mesh size was larger than that of the beads. When we mixed

14 the beads with the viscous liquid of Phos-cycC $\mathbf{C}_{6}$, the fluorescent beads and supramolecular

15 fibres could be distinctly visualised, as shown in Figure 4a. Supplementary movie 1 shows

16 representative examples using $300 \mathrm{~nm}$ beads, in which the free Brownian motion of the beads

17 in the Phos-cycC ${ }_{6}$ sol almost stopped $3 \mathrm{~h}$ after the addition of BAP. Figure $4 \mathrm{~b}$ shows two

18 snapshot images of the $300 \mathrm{~nm}$ beads in the Phos-cycC $_{6}$ matrix with a 5 min interval of

19 CLSM observations. The positions of almost all the beads changed after 5 min without BAP

20 treatment, whereas the positions of the beads did not change 5 min after the BAP treatment

21 (Figure 4b). These CLSM images indicated that beads $70 \mathrm{~nm}$ to $500 \mathrm{~nm}$ in diameter moved

22 freely in the viscous liquid of the $\mathbf{P h o s}_{-\mathbf{c y c}} \mathbf{C}_{\mathbf{6}}$ fibre network $(0.15 \mathrm{wt} \%)$ whereas the $800 \mathrm{~nm}$

23 beads did not move. Therefore, the mesh size of the $\mathbf{P h o s}-\mathbf{c y c} \mathbf{C}_{\mathbf{6}}$ viscous liquid was between 
1500 and $800 \mathrm{~nm}$ (Figure 4c). In contrast, only $70 \mathrm{~nm}$ beads moved freely and beads larger

2 than $200 \mathrm{~nm}$ in diameter almost stopped moving after BAP treatment. Figure 4c shows that

3 the bead size critical for turning the Brownian motion on and off shifted from $500-800$ to

4 200-300 nm after the addition of BAP, which implied that the mesh size became smaller.

5 These results consistently demonstrated that a dense cross-linked fibre network was obtained

6 by BAP treatment, which facilitated gelation of the viscous liquid of Phos-cycC 6 .

7 
1 Bidirectional tuning of rheological properties and protein release profiles of the SDN

2 hydrogel

3 If the two gelators are co-assembled upon mixing, the original properties of these fibres may

4 interfere, possibly resulting in hybrid fibres showing properties different from the sum of the

5 original ones. In contrast, it is reasonable to expect the programmable construction of a

6 hybrid hydrogel that retains distinct stimulus-responsiveness upon mixing of two fibres if

7 these are orthogonally self-sorted (a possible scenario shown in Figure 5a).

According to this scenario, we prepared a mixed-hydrogel comprising NPmoc-F(4-F)F

9 and Phos-cycC $_{6}$ and monitored the stimulus responses. The two-component SDN hydrogel

10 changed to a viscous liquid within 10 min after the addition of $\mathrm{Na}_{2} \mathrm{~S}_{2} \mathrm{O}_{4}$ whereas the addition

11 of BAP toughened the hydrogel (Figure 5b). As shown in Figure 5c, the rheological data

12 quantitatively supported the behaviours observed by the naked eye. The $G^{\prime}$ and $G^{\prime \prime}$ values of

13 the SDN hydrogel were respectively 187 and $60 \mathrm{~Pa}$, without stimuli, which increased to 1450

14 and $336 \mathrm{~Pa}$ (7.8-fold for $G^{\prime}$, 5.6-fold for $G^{\prime \prime}$ ), respectively, after BAP treatment. The

15 reinforcement may have been caused by increase in the number of cross-linking points and/or

16 pseudo-cross-linking points between fibres in the SDN hydrogel. ${ }^{41}$ In sharp contrast, these

17 values decreased almost 100 -fold to $2 \mathrm{~Pa}$ for $G^{\prime}$ and $1 \mathrm{~Pa}$ for $G^{\prime \prime}$ after the addition of $\mathrm{Na}_{2} \mathrm{~S}_{2} \mathrm{O}_{4}$.

18 Complete rheological characterisations of the SDN hydrogels are shown in Supplementary

19 Figures 21-25. SDN hydrogels with the different ratios (NPmoc-F(4-F)F: 0.4 wt\%,

20 Phos-cycC 6 : $0.4 \mathrm{wt} \%$ ) also showed the same bidirectional control of rheological properties

21 (Supplementary Figure 26). These data clearly demonstrated that the present two-component

hydrogel prepared by simple mixing exhibited bidirectional rheological response depending

23 on the applied stimulus. The HPLC analysis of this SDN hydrogel confirmed that 
1 NPmoc-F(4-F)F reacted with $\mathrm{Na}_{2} \mathrm{~S}_{2} \mathrm{O}_{4}$, whereas it was unaffected by the BAP treatment (the

2 residual ratio was $>95 \%$ ) (Figure 5d, Supplementary Figure 27). These were in agreement

3 with the results of the CD spectral changes (Supplementary Figure 28). Conversely,

4 Phos-cycC $\mathrm{C}_{6}$ remained intact after treatment with $\mathrm{Na}_{2} \mathrm{~S}_{2} \mathrm{O}_{4}$ and was able to react with BAP

5 (Figure 5d, Supplementary Figure 27). The CD spectral data agreed with the theoretical sum

6 of the BAP-treated single-component Phos-cycC 6 and NPmoc-F(4-F)F (Supplementary

7 Figure 28). Moreover, CLSM images of the resultant hydrogels clearly showed orthogonal

8 fibrous structures (Supplementary Figure 29). This strongly suggested that the original

9 self-sorting fibres retained their orthogonality even after BAP treatment.

10 On the basis of this unique rheological property, we subsequently applied this SDN

11 hydrogel as a matrix for controlled protein release. After a protein (immunoglobulin $\mathrm{G}(\mathrm{IgG})$,

12 myoglobin $(\mathrm{Mb})$, or concanavalin A (ConA)) was encapsulated in the SDN hydrogel, a

13 stimulus $\left(\mathrm{Na}_{2} \mathrm{~S}_{2} \mathrm{O}_{4}, \mathrm{BAP}\right.$, or none) was applied to the gel matrix, and proteins release was

14 evaluated using SDS-PAGE of the supernatant solution. As shown in Figure 5e and

15 Supplementary Figure $30,40 \pm 12 \%$ of the embedded IgG was slowly released over $10 \mathrm{~h}$

16 without stimulus. Interestingly, $\mathrm{IgG}$ release was enhanced by $\mathrm{Na}_{2} \mathrm{~S}_{2} \mathrm{O}_{4}$ addition $(87 \pm 19 \%)$,

17 but suppressed by BAP addition $(14 \pm 2 \%)$. Bidirectionally tuned release profiles are also

18 shown for other proteins ( $\mathrm{Mb}$ and ConA, Figure 5e and Supplementary Figure 31 and 32). 


\section{Adaptive response of the SDN hydrogel depending on the order of applied stimuli}

2 We further demonstrated that the bidirectional rheological properties of this SDN hydrogel

3 can produce unprecedented gel-sol transition behaviour depending on the order in which the

4 two stimuli were applied in a stepwise manner (Figure 6a). In these experiments, we applied

5 two stimuli $\left(\mathrm{Na}_{2} \mathrm{~S}_{2} \mathrm{O}_{4}\right.$ and BAP) to the SDN hydrogel in the two different sequences $\left(1^{\text {st }}\right.$

$6 \mathrm{Na}_{2} \mathrm{~S}_{2} \mathrm{O}_{4} / 2^{\text {nd }}$ BAP (route A) or $1^{\text {st }} \mathrm{BAP} / 2^{\text {nd }} \mathrm{Na}_{2} \mathrm{~S}_{2} \mathrm{O}_{4}$ (route B)) and observed the macroscopic

7 changes. As shown in Figure 6b, the SDN hydrogel changed to a viscous liquid when

$8 \mathrm{Na}_{2} \mathrm{~S}_{2} \mathrm{O}_{4}$ was added first, and the resultant viscous liquid recovered to a hydrogel after

9 subsequent BAP treatment. When the input order was reversed (route B), the hydrogel state

10 remained after each step without appearance of the sol state (Figure 6c). The SDN hydrogel

11 exhibited two distinct response patterns, i.e., the gel-sol-gel for route A and the gel-gel-gel

12 for route $\mathrm{B}$, depending on the order in which the stimuli were applied. This stimulus-order

13 dependent gel-sol change allowed us to encapsulate fluorescent nanobeads under particular

14 conditions. Nanobeads dispersed in aqueous solution were poured on the surface of SDN hydrogels followed by the addition of $\mathrm{Na}_{2} \mathrm{~S}_{2} \mathrm{O}_{4}$. After $30 \mathrm{~min}$, the gel was destroyed owing to

16 decomposition of the NPmoc-F(4-F)F fibre network, and thus, the beads got immersed in the

17 resultant viscous liquid. The subsequent addition of BAP converted the viscous liquid to a

18 hydrogel again; the nanobeads were encapsulated within the gel matrix (Figure 6f, upper).

19 Conversely, in route $\mathrm{B}$, the gel did not change during any of the stages; therefore, the

20 nanobeads could not be encapsulated (Figure 6f, bottom). Even though the same two stimuli

21 were applied overall, the fluorescent SDN hydrogel encapsulated nanobeads only by the operation of the $1^{\text {st }} \mathrm{Na}_{2} \mathrm{~S}_{2} \mathrm{O}_{4} / 2^{\text {nd }}$ BAP stimuli. Therefore, it was concluded that our SDN hydrogel was an adaptive soft-materials capable of recognising the order of input stimuli. ${ }^{28,29}$ 
This unique macroscopic gel/sol transition was carefully investigated by HPLC

2 analysis, CD spectroscopy and CLSM observation of the Brownian motion of nanobeads.

3 Time-dependent product analyses by HPLC quantitatively showed that the two gelators

4 underwent distinct chemical conversion in each step of the two different routes

5 (Supplementary Figure 33), which corresponded well with the destruction and entanglement

6 of self-sorting supramolecular nanofibres. The CD spectra of SDN hydrogels after the

7 addition of stimuli in different orders were almost identical, which indicated that the final

8 structure was identical irrespective of the stimulus order (Supplementary Figure 34). We also

9 used CLSM to trace the Brownian motion of the $300 \mathrm{~nm}$ (diameter) fluorescent beads

10 embedded in the SDN gel matrix (Figure 6d, e, Supplementary movies 2-5). Without stimuli,

11 almost none of the beads moved. In route A, they started to move randomly after the addition

12 of $\mathrm{Na}_{2} \mathrm{~S}_{2} \mathrm{O}_{4}$ (Figure 6d, Supplementary movie 2), clearly indicating that the mesh size

13 increased to above $300 \mathrm{~nm}$. Subsequent BAP treatment caused the suppression (almost

14 stoppage) of Brownian motion of the beads, implying that the mesh size reduced to below

$15300 \mathrm{~nm}$ again (Figure 6d, Supplementary movie 3). In route B, all of the fluorescent beads

16 were always stationary, indicating that the mesh size was smaller than $300 \mathrm{~nm}$ during all

17 three steps (Figure 6e, Supplementary movies 4, 5). These results revealed that the mesh size

18 was modulated by the sequence in which stimuli were applied. 


\section{Conclusions}

2 We have shown a self-sorting supramolecular nanofibre pair, prepared on the basis of the

3 different driving forces for self-assembly of each component. The distinct nanofibres allowed

4 preparation of a rationally designed self-sorting hydrogel with bidirectional rheological and

5 protein release profile changes in response to two orthogonal stimuli. Moreover, the hydrogel

6 exhibited an adaptive gel-sol response by discriminating the order of the stimuli applied. Our

7 hydrogel can be programmed with a desired function, highlighting the utility of self-sorting

8 systems for the bottom-up design of multicomponent intelligent soft materials for a variety of

9 applications including therapy, diagnosis, drug delivery, and regenerative medicine. 


\section{$1 \quad$ References}

2 1. Alberts, B. et al. Molecular Biology of the Cell 5th edn, 965-1052 (Garland Science, $32008)$

4 2. Parry, D. A. D. \& Squire, J. M. Fibrous proteins: structures and mechanisms (Springer, 5 2017).

6 3. Aida, T., Meijer, E. W. \& Stupp, S. I. Functional supramolecular polymers. Science 335, 813-817 (2012).

4. Webber, M. J., Appel, E. A., Meijer, E. W. \& Langer, R. Supramolecular biomaterials. Nat. Mater. 15, 13-26 (2016).

5. Safont-Sempere, M. M., Fernández, G. \& Würthner, F. Self-sorting phenomena in complex supramolecular systems. Chem. Rev. 111, 5784-5814 (2011).

6. Adler-Abramovich, L. \& Gazit, E. The physical properties of supramolecular peptide assemblies: from building block association to technological applications. Chem. Soc. Rev. 43, 6881-6893 (2014).

7. Adhikari, B., Nanda, J. \& Banerjee, A. Multicomponent hydrogels from enantiomeric amino acid derivatives: helical nanofibers, handedness and self-sorting. Soft Matter 7, 8913-8922 (2011).

8. Buerkle L. E. \& Rowan, S. J. Supramolecluar gels formed from multi-component low molecular weight species. Chem. Soc. Rev. 41, 6089-6102 (2012).

9. Shigemitsu, H. \& Hamachi, I. Supramolecular assemblies responsive to biomolecules toward biological applications. Chem. Asian J. 10, 2026-2038 (2015).

10. Molla, M. R., Das, A. \& Ghosh, S. Self-sorted assembly in a mixture of donor and acceptor chromophores. Chem. Eur. J. 16, 10084-10093 (2010). 
1 11. Smith, M. M. \& Smith, D. K. Self-sorting multi-gelator gels-mixing and ageing effects in thermally addressable supramolecular soft nanomaterials. Soft Matter 7, 4856-4860 (2011).

12. Hirst, A. R., Eschder, B., Miravet, J. F. \& Smith, D. K. High-tech applications of self-assembling supramolecular nanostructured gel-phase materials: from regenerative medicine to electronic devices. Angew. Chem. Int. Ed. 47, 8002-8018 (2008).

13. Raeburn, J. \& Adams, D. J. Multicomponent low molecular weight gelators. Chem. Commun. 51, 5170-5180 (2015).

14. Görl, D., Zhang, X., Stepanenko, V. \& Würthner, F. Supramolecular block copolymers by kinetically controlled co-self-assembly of planar and core-twisted perylene bisimides. Nat. Commun. 6, 7009 (2015).

15. Heeres, A. et al. Orthogonal self-assembly of low molecular weight hydrogelators and surfactants. J. Am. Chem. Soc. 125, 14252-14253 (2003).

16. Li, C. et al, Responsive double network hydrogels of interpenetrating DNA and CB[8] Host-Guest supramolecular systems. Adv. Mater. 27, 3298-3304 (2015).

17. Colquhoun, C. et al. The effect of self-sorting and co-assembly on the mechanical properties of low molecular weight hydrogels. Nanoscale 6, 13719-13725 (2014).

18. Pal, A., Besenius, P. \& Sijbesma, R. P. Self-sorting in rodlike micelles of chiral bisurea bolaamphiphiles. J. Am. Chem. Soc. 133, 12987-12989 (2011).

19. Draper, E. R., Jonathan, R. L., Wallace, M., Jäckel, F., Cowan, A. J., Adams, D. J. Self-sorted photoconductive xerogels. Chem. Sci. 7, 6499-6505 (2016). 
1 20. Boekhoven, J., Koot, M., Wezendonk, T. A., Eelkema, R. \& van Esch, J. H. A self assembled delivery platform with post-production tunable release rate. J. Am. Chem. Soc. 134, 12908-12911 (2012).

21. Morris, K. L. et al. Chemically programmed self-sorting of gelator networks. Nat. Commun. 4, 1480 (2013).

22. Draper, E. R., Eden, E. G. B., McDonald, T. O. \& Adams, D. J. Spatially resolved multicomponent gels. Nat. Chem. 7, 848-852 (2015).

23. Cornwell D. J., Daubney O. J. \& Smith D. K. Photopatterned multidomain gels: multi-component self-assembled hydrogels based on partially self-sorting 1,3:2,4-Dibenzylidene- $d$-sorbitol derivatives. J. Am. Chem. Soc. 137, 15486-15492 (2015).

24. Singh, N. et al. Tandem reactions in self-sorted catalytic molecular hydrogels. Chem. Sci. 7, 5568-5572 (2016).

25. Sugiyasu, K., Kawano, S.-i., Fujita, N. \& Shinkai, S. Self-sorting organogels with p-n heterojunction points. Chem. Mater. 20, 2863-2865 (2008).

26. Prasanthkumar, S. et al. Organic donor-acceptor assemblies form coaxial $\mathrm{p}-\mathrm{n}$ heterojunctions with high photoconductivity. Angew. Chem. Int. Ed. 54, 946-950 (2015).

27. Cornwell, D. J. \& Smith, D. K. Expanding the scope of gels - combining polymers with low-molecular weight gelators to yield modified self-assembling smart materials with high-tech applications. Mater. Horiz. 2, 279-293 (2015).

28. Ikeda, M. et al. Installing logic-gate responses to a variety of biological substances in supramolecular hydrogel-enzyme hybrids. Nat. Chem. 6, 511-518 (2014). 
1 29. Komatsu, H. et al. Supramolecular hydrogel exhibiting four basic logic gate functions to fine-tune substance release. J. Am. Chem. Soc. 131, 5580-5585 (2009).

30. S. Onogi et al. In situ real-time imaging of self-sorted supramolecular nanofibres. Nat. Chem. 8, 743-752 (2016).

31. Boekhoven, J. et al. Bio-inspired supramolecular materials by orthogonal self-assembly of hydrogelators and phospholipids. Chem. Sci. 7, 6021-6031 (2016).

32. Ikeda, M., Tanida, T., Yoshii, T. \& Hamachi, I. Rational molecular design of stimulus-responsive supramolecular hydrogel based on dipeptides. Adv. Mater. 23, 2819-2822 (2011).

33. Shigemitsu, H. et al. Preparation of supramolecular hydrogel-enzyme hybrids exhibiting biomolecule-responsive gel degradation. Nat. Protoc. 11, 1744-1756 (2016).

34. Kiyonaka, S., Sugiyasu, K., Shinkai, S. \& Hamachi, I. First thermally responsive supramolecular polymer based on glycosylated amino acid. J. Am. Chem. Soc. 124, 10954-10955 (2002).

35. Dunn, K.W., Kamocka, M. M. \& McDonald, J. H. A practical guide to evaluating colocalization in biological microscopy. Am. J. Physiol. Cell Physiol. 300, C723-C742 (2011).

36. Tamaru, S.-i. et al. Fluidic supramolecular nano- and microfibres as molecular rails for regulated movement of nanosubstances. Nat. Commun. 1, 20 (2010).

37. Thompson, N. L., Burghardt, T. P. \& Axelrod, D. Measuring surface dynamics of biomolecules by total internal reflection fluorescence with photobleaching recovery or correlation spectroscopy. Biophys. J. 33, 435-454 (1981). 
1 38. Jayaraman, K. et al. Observing capillarity in hydrophobic silica nanotubes. J. Am. Chem.

2

3

4

5

6

7

8

9

10

41. Shams Es-haghi, S. \& Weiss, R. A. Fabrication of tough hydrogels from chemically Soc. 127, 17385-17392 (2005).

39. Matsumoto, S. et al. Photo gel-sol/sol-gel transition and its patterning of a supramolecular hydrogel as stimuli-responsive biomaterials. Chem. Eur. J. 14, 39773986 (2008).

40. Yoshii, T., Ikeda, M. \& Hamachi, I., Two-photon-responsive supramolecular hydrogel for controlling materials motion in micrometer space. Angew. Chem. Int. Ed. 53, 72647267 (2014). cross-linked multiple neutral networks. Macromolecules, 49, 8980-8987 (2016). 


\section{Acknowledgements}

2 The authors thank M. Suginome and Y. Nagata (Kyoto University) for CD spectra

3 measurements, N. Yamada (Kyoto Insititute of Technology) for their kind support with

4 rheological measurements, and Y. Sato (Carl Zeiss Microimaging Co.) for CLSM imaging

5 with Airyscan unit. H. S. acknowledges JSPS Research Fellowships for Young Scientists

6 (16J10716). The authors acknowledge financial support from The Mitsubishi foundation.

7

\section{Author contributions}

9 H. S. and I. H. conceived the project and designed the experiments. H. S., T. F., and W. T.

10 performed all the experiments. H. S., S. M. and K. U. analyzed rheological properties. The

11 paper was written by H. S., R. K., and I. H. and edited by all the co-authors.

12

\section{Competing financial interests}

14 The authors declare no competing financial interests. 


\section{Figure Captions}

(a)

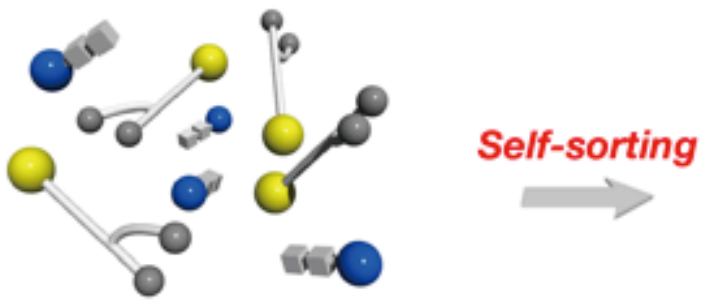

Peptide- and lipid-type gelators

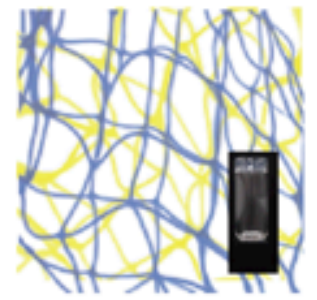

Self-sorting double network (SDN) hydrogel (b) Peptide-type gelators<smiles>[R]c1ccc(CC(NC(=O)OCc2ccc([N+](=O)[O-])cc2)C(=O)N[C@@H](Cc2ccccc2)C(=O)O)cc1</smiles>

NPmoc-FF: $\mathrm{R}=\mathrm{H}$

NPmoc-F(4-F)F: $R=F$
Lipid-type gelator<smiles>COP(=O)([O-])OCCCCCCCCNC(=O)/C=C/C(=O)N[C@@H](CCC(=O)OC1CCCCC1)C(=O)OC1CCCCC1</smiles>

Phos-cycC 6

Fluorescence probe for NPmoc-FF derivatives

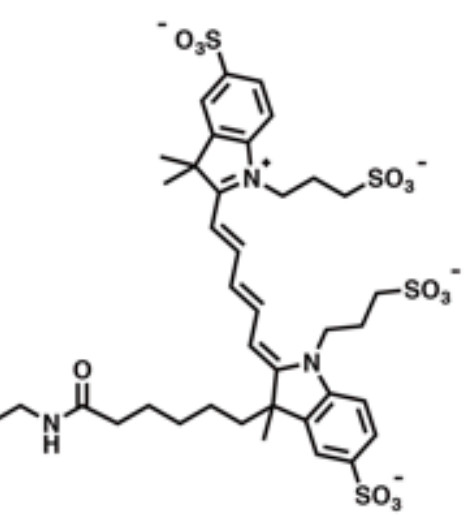

NP-Alexa647

Fluorescence probe for Phos-cycC 6<smiles>O=C(/C=C/C(=O)N[C@@H](CCC(=O)OC1CCCCC1)C(=O)OC1CCCCC1)NCCCCCCCCNc1ccc([N+](=O)[O-])c2nonc12</smiles>

NBD-cycC 6 
1 Figure 1. Schematic representation of formation of a self-sorting double network (SDN) 2 hydrogel, and molecular structures of hydrogelators and fluorescence probes. (a) Two 3 low molecular weight peptide- and lipid-type hydrogelators orthogonally assemble into two 4 distinct supramolecular nanofibres and form a SDN hydrogel. The SDN hydrogel shows the 5 adaptive functions such as bidirectional control of rheological property and release rate of 6 encapsulated molecules by specific external stimuli. The peptide- and lipid-type 7 hydrogelators are described as schematic blue and yellow molecules, respectively. The blue 8 and yellow networks shown in the right indicate nanofibres consisting of self-assembled 9 peptide- and lipid-type hydrogelators, respectively. (b) Chemical structures of peptide- and 10 lipid-type gelators (NPmoc-FF, NPmoc-F(4-F)F, and $\mathbf{P h o s}-\mathbf{c y c C}_{6}$ ) and fluorescence probes 11 (NP-Alexa647 and NBD-cycC $_{6}$ ) which selectively stain self-sorting supramolecular 12 nanofibres and enable in situ CLSM imaging.

13

14

15

16 
(a) NPmoc-F(4-F)F

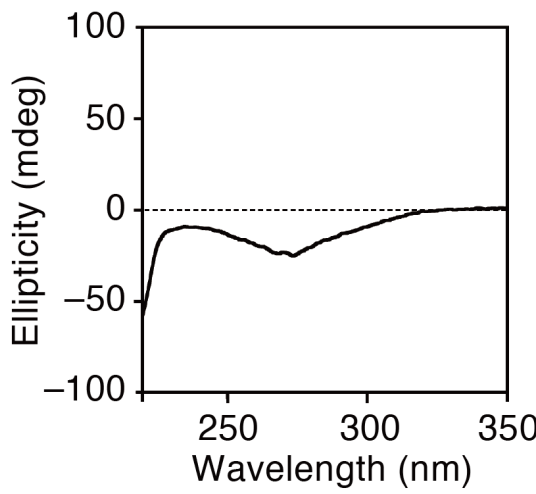

(d) NP-Alexa647

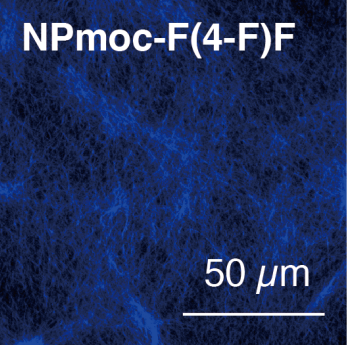

(b) Phos-cycC

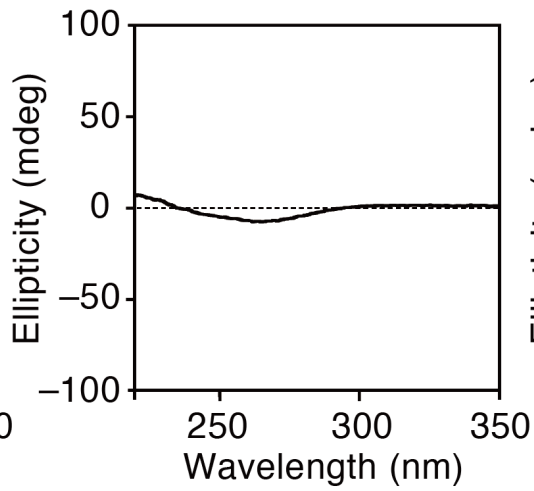

(c) Mixture

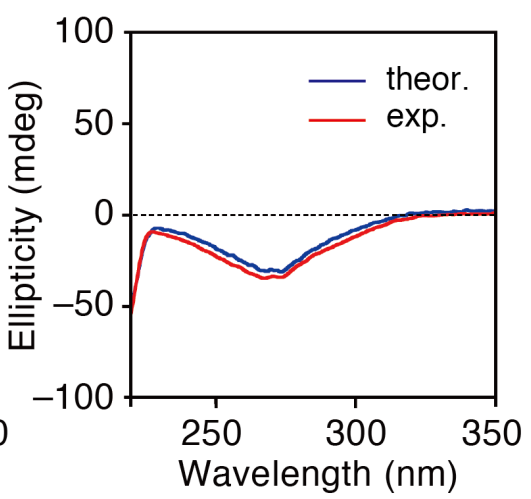

(e) NBD-cycC 6
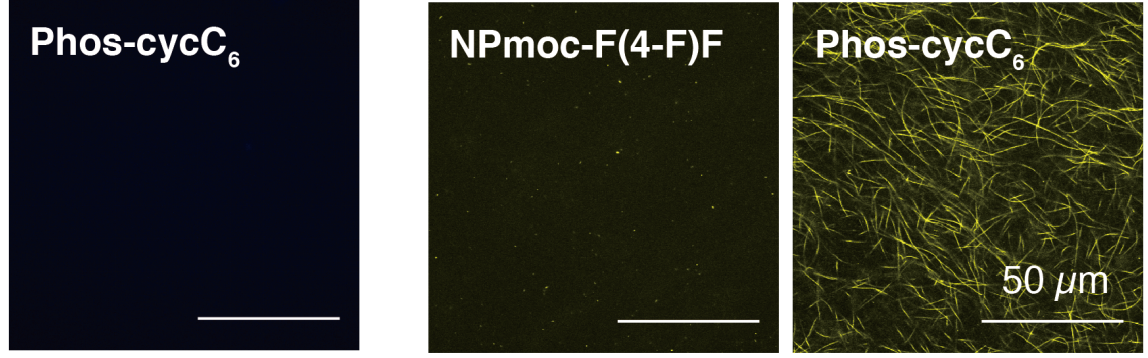

(f) Mixture/Airyscan
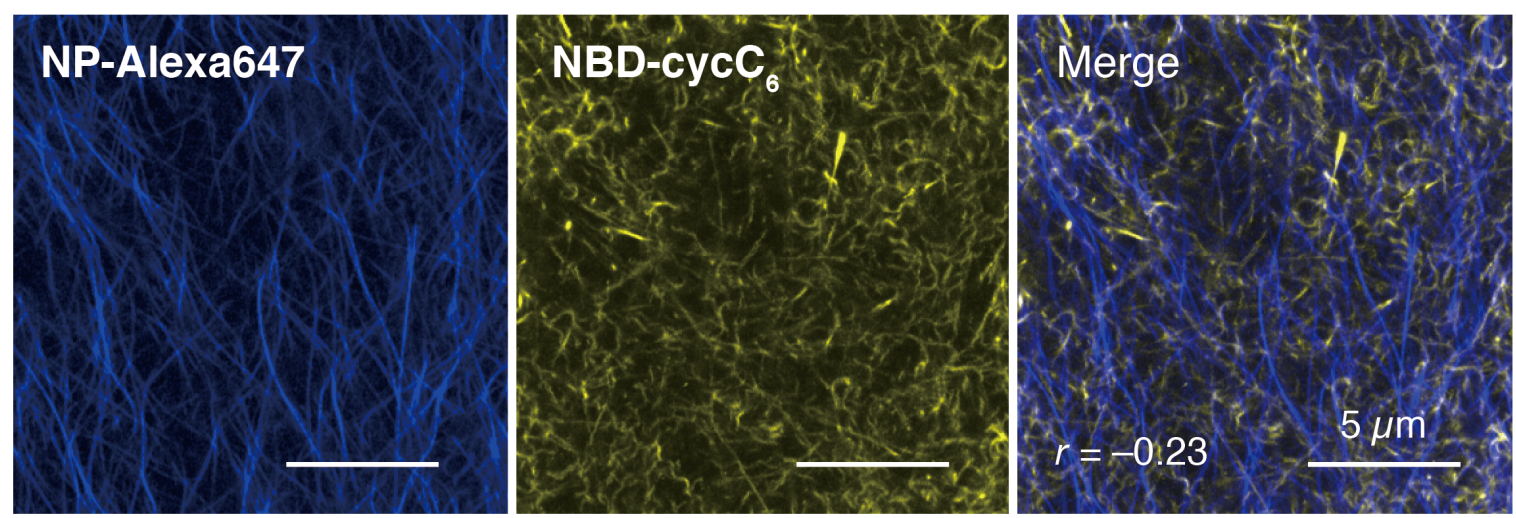
1 Figure 2. Evaluation for self-sorting of NPmoc-F(4-F)F and Phos-cycC 6 . (a-c) CD 2 spectra of NPmoc-F(4-F)F (a), Phos-cycC $_{6}$ (b), and a mixture of NPmoc-F(4-F)F and 3 Phos-cycC $_{6}$ (c). The blue and red lines in (c) show theoretical and experimental CD spectra, 4 respectively. The theoretical CD spectrum is a simple sum of the CD spectra of 5 NPmoc-F(4-F)F and Phos-cycC $\mathbf{6}_{6}((\mathrm{a})$ and (b)). The HT data is shown in Supplementary 6 Figures 5 and 6. CD spectra measured under different conditions also shown in 7 Supplementary Figure 7. (d, e) CLSM images of NPmoc-F(4-F)F and Phos-cycC $_{6}$ 8 supramolecular nanofibres in the presence of NP-Alexa647 (d) and NBD-cycC $_{6}$ (e). (f) 9 High-resolution CLSM (with Airyscan unit) images of self-sorting NPmoc-F(4-F)F and 10 Phos-cycC $_{6}$ fibres stained by NP-Alexa647 and NBD-cycC $_{6}$, respectively. The left, middle, 11 and right images show NPmoc-F(4-F)F, Phos-cycC $\mathbf{C}_{6}$ fibres and the merged images, 12 respectively. The number in the merged image is the Pearson correlation coefficient value $(r)$. 13 Scale bar: $5 \mu \mathrm{m}$. Conditions: $[\mathbf{N P m o c - F ( 4 - F ) F}]=0.40 \mathrm{wt} \%(7.9 \mathrm{mM}),\left[\right.$ Phos-cycC $\left._{6}\right]=0.15$ $14 \mathrm{wt} \%(2.4 \mathrm{mM}),[\mathbf{N P}-A l e x a 647]=4.0 \mu \mathrm{M},[$ NBD-cycC 6$]=20 \mu \mathrm{M}$ in $100 \mathrm{mM}$ HEPES buffer 15 (pH 8.0), rt. 
(a)

a)

NPmoc-F(4-F)F

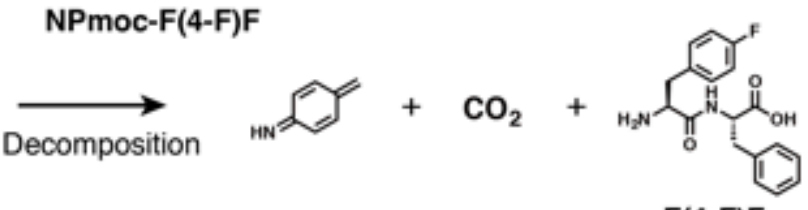

$F(4-F) F$ (b)

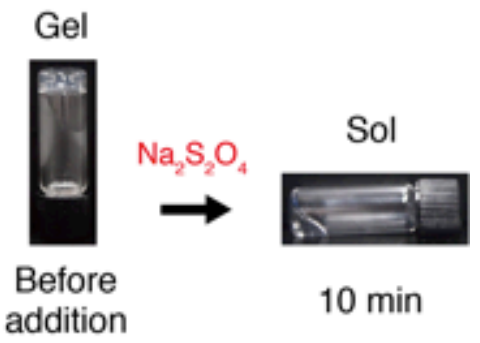

(c)

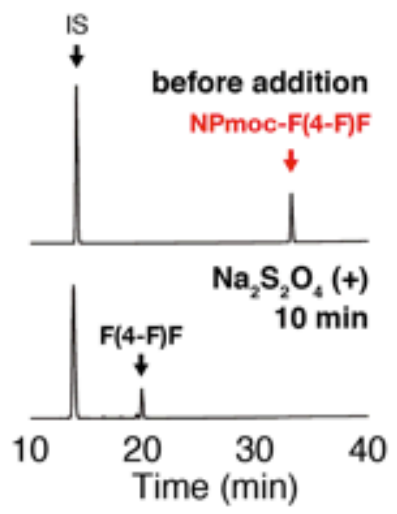

(d)

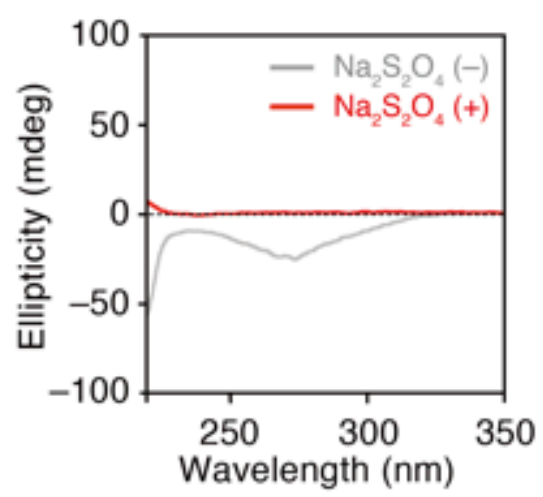

(e)

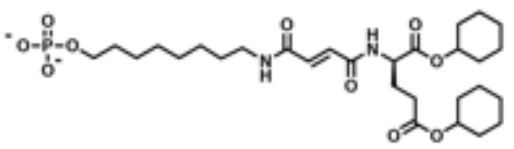

Phos-cycC 6

(f)

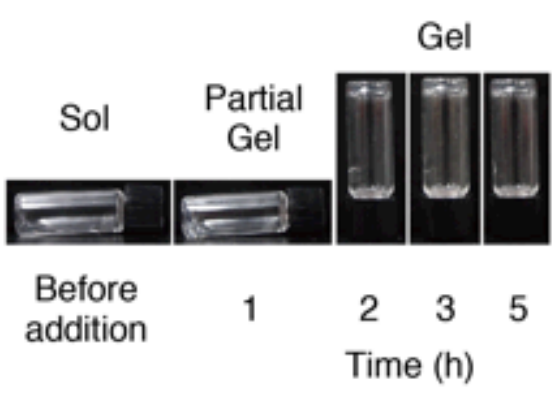

(h)

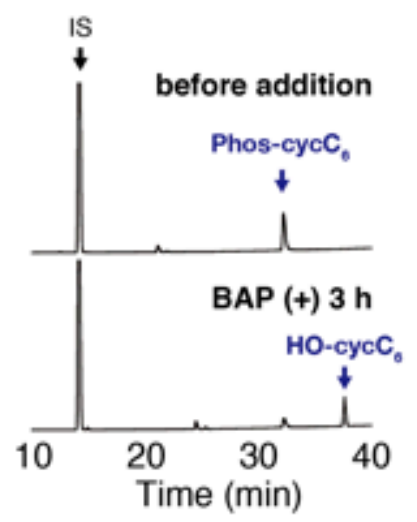

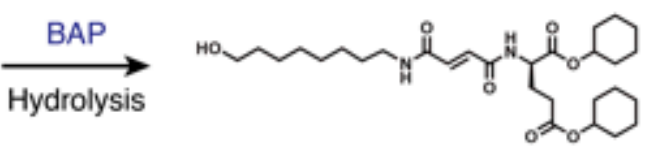

HO- -ycC $_{6}$

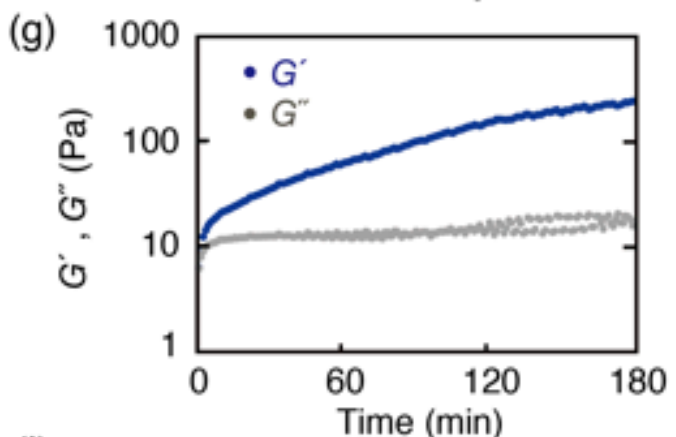

(i)

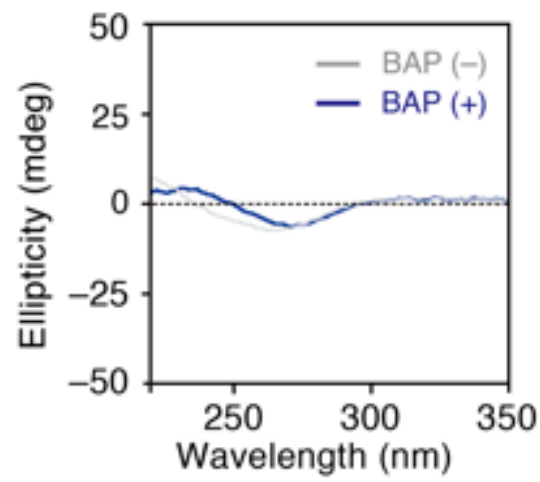


1 Figure 3. Stimuli responsiveness of NPmoc-F(4-F)F and Phos-cycC 6 ((a)-(d): 2 NPmoc-F(4-F)F, (e)-(i): Phos-cycC 6 ). (a) Chemical reaction between NPmoc-F(4-F)F and $3 \mathrm{Na}_{2} \mathrm{~S}_{2} \mathrm{O}_{4}$. The nitro group is reduced by $\mathrm{Na}_{2} \mathrm{~S}_{2} \mathrm{O}_{4}$ and the generated aniline derivative 4 spontaneously decomposes. (b) Optical photos of NPmoc-F(4-F)F gel and the sol after 5 addition of $\mathrm{Na}_{2} \mathrm{~S}_{2} \mathrm{O}_{4}(10 \mathrm{~min})$. (c) HPLC charts of NPmoc-F(4-F)F gel before and after 6 addition of $\mathrm{Na}_{2} \mathrm{~S}_{2} \mathrm{O}_{4}(10 \mathrm{~min})$. (d) CD spectra of NPmoc-F(4-F)F gel (gray) and the sol 7 obtained from NPmoc-F(4-F)F gel by addition of $\mathrm{Na}_{2} \mathrm{~S}_{2} \mathrm{O}_{4}$ (red). Conditions: $8 \quad[\mathbf{N P m o c}-\mathbf{F}(4-\mathbf{F}) \mathbf{F}]=0.40 \mathrm{wt} \%(7.9 \mathrm{mM}),\left[\mathrm{Na}_{2} \mathrm{~S}_{2} \mathrm{O}_{4}\right]=79 \mathrm{mM}(10$ eq. for NPmoc-F(4-F)F $)$ 9 in $100 \mathrm{mM}$ HEPES buffer $(\mathrm{pH} 8.0) . V_{\text {gel }} / V_{\mathrm{Na}_{2} \mathrm{~S}_{2} \mathrm{O}_{4} \text { aq. }}=10: 1,25^{\circ} \mathrm{C}, 10 \mathrm{~min}$. (e) Chemical 10 reaction between Phos-cycC $_{6}$ and BAP. (f) Optical photos of Phos-cycC $_{6}$ solution after 11 addition of BAP. (g) Time-course of the rheological properties of the Phos-cycC $_{6}$ solution 12 after addition of BAP (0-180 min). (h) HPLC charts of Phos-cycC $\mathbf{C}_{6}$ solution or gel after 13 addition of BAP (3 h). (i) CD spectra of Phos-cyc $\mathbf{C}_{6}$ solution and the gel obtained by 14 addition of BAP. Conditions: [Phos-cycC $\left.\mathbf{C}_{6}\right]=0.15 \mathrm{wt} \%(2.4 \mathrm{mM}),[\mathrm{BAP}]=0.05 \mathrm{U} / \mu \mathrm{L}$ in $15100 \mathrm{mM}$ HEPES buffer $(\mathrm{pH} 8.0) . V_{\text {gel }} / V_{\mathrm{BAP}}=10: 1,25^{\circ} \mathrm{C}, 300 \mathrm{~min}$. IS in figures (c) and (h) 16 mean internal standard (terephthalic acid). 
(a)
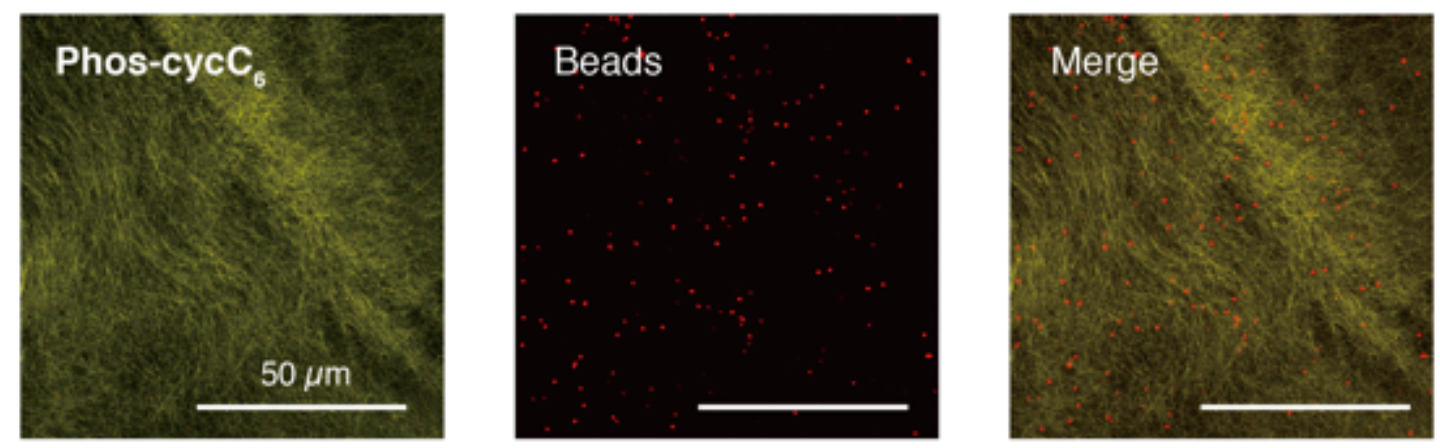

(b) $\mathrm{BAP}(-)$
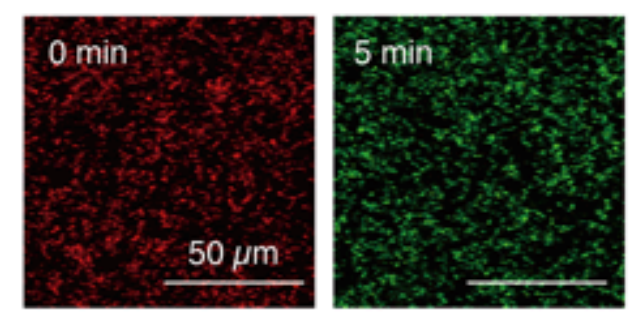

(c)

$$
\operatorname{BAP}(+), 3 \mathrm{~h}
$$
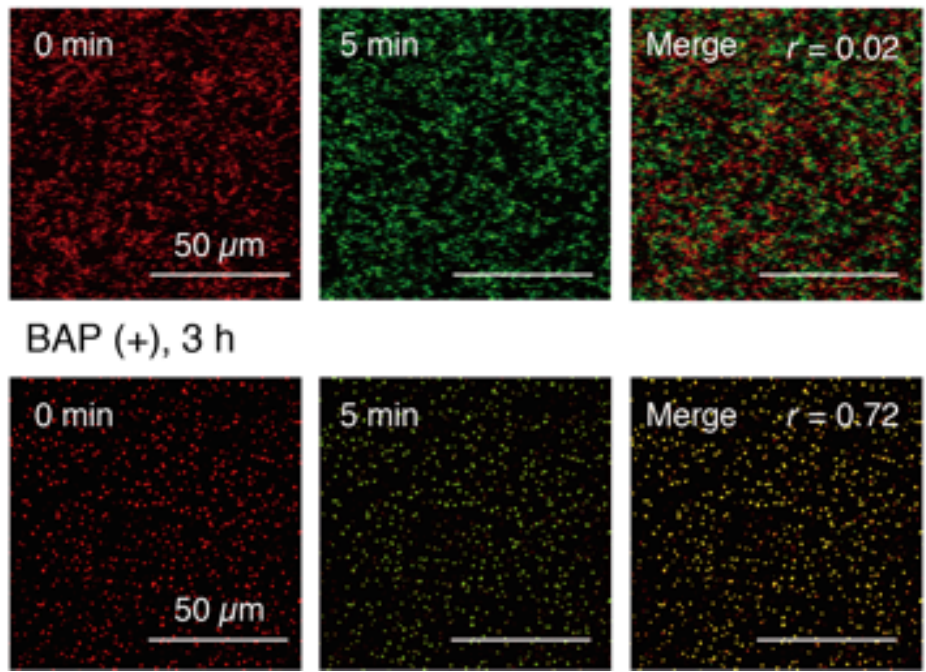

\begin{tabular}{ccc}
\hline $\begin{array}{c}\text { Beads } \\
\text { diameter } \\
(\mathrm{nm})\end{array}$ & \multicolumn{2}{c}{ BAP } \\
\hline 70 & ON & ON \\
200 & ON & ON / OFF \\
300 & ON & OFF \\
500 & ON & OFF \\
800 & OFF & OFF \\
\hline
\end{tabular}


1 Figure 4. Brownian motions of beads in Phos-cycC $_{6}$ sol or gel generated by BAP 2 treatment. (a) CLSM images of the mixture of $\mathbf{P h o s}_{-\mathbf{c y c}} \mathbf{C}_{6}$ and fluorescent beads $(800 \mathrm{~nm})$ 3 in buffer solution. The right, middle, and left images show Phos-cyc $\mathbf{C}_{6}$ fibres stained with $4 \quad$ NBD-cycC $_{6}$, fluorescent beads and the merged image, respectively. Scale bar: $50 \mu \mathrm{m}$. (b) 5 CLSM images of the Brownian motions of beads (diameter: $300 \mathrm{~nm}$ ) in Phos-cycC $\mathbf{C}_{\mathbf{6}}$ sol and 6 gel. The red (left) and green (middle) CLSM images show the positions of the fluorescence 7 beads at 0 and $5 \mathrm{~min}$ after starting the observation. The right images show the combined 8 images. In the images, the yellow beads indicate that the positions of the beads did not 9 change at 0 and 5 min after starting the observations. The numbers in the images represent 10 Pearson correlation coefficient $(r)$. The movie of the Brownian motions of the beads can be 11 seen in supplementary movie S1. Scale bar: $25 \mu \mathrm{m}$. (c) Brownian motions of beads (diameter: $1270-800 \mathrm{~nm})$ in Phos-cycC $_{6}$ solution and the gel after addition of BAP $(3 \mathrm{~h})$. Conditions: $13\left[\right.$ Phos-cycC $\left._{6}\right]=0.15 \mathrm{wt} \%(2.4 \mathrm{mM}),[$ NBD-cycC 6$]=20 \mu \mathrm{M},[\mathrm{BAP}]=0.05 \mathrm{U} / \mu \mathrm{L}$. 14 [Fluorescence nanobeads $(300 \mathrm{~nm})]=0.05 \mathrm{mg} / \mathrm{mL}$ in $100 \mathrm{mM}$ HEPES buffer $(\mathrm{pH} 8.0)$. $15 V_{\mathrm{gel}} / V_{\mathrm{BAP}}=10: 1,25^{\circ} \mathrm{C}, 3 \mathrm{~h}$. 
(a)
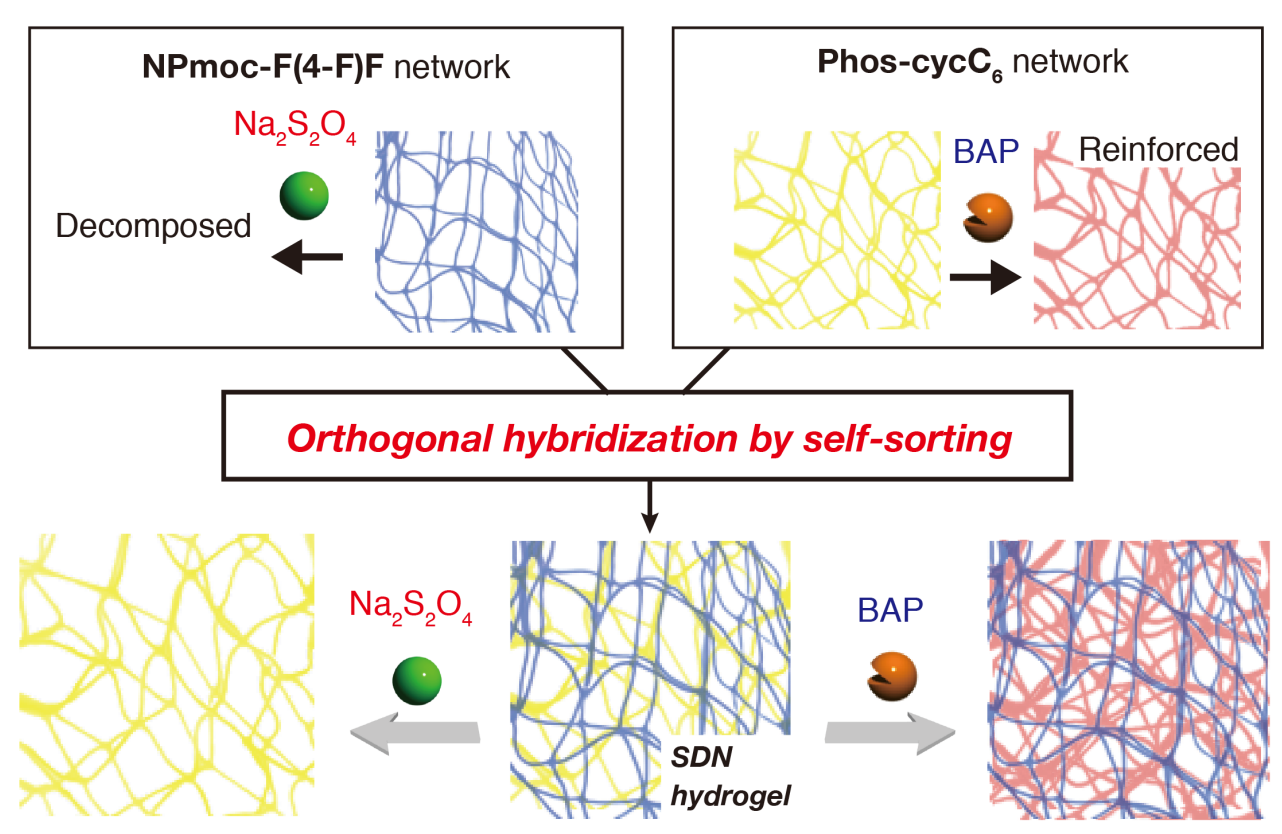

Phos-cycC 6

Lower

Higher

(b)

(c)

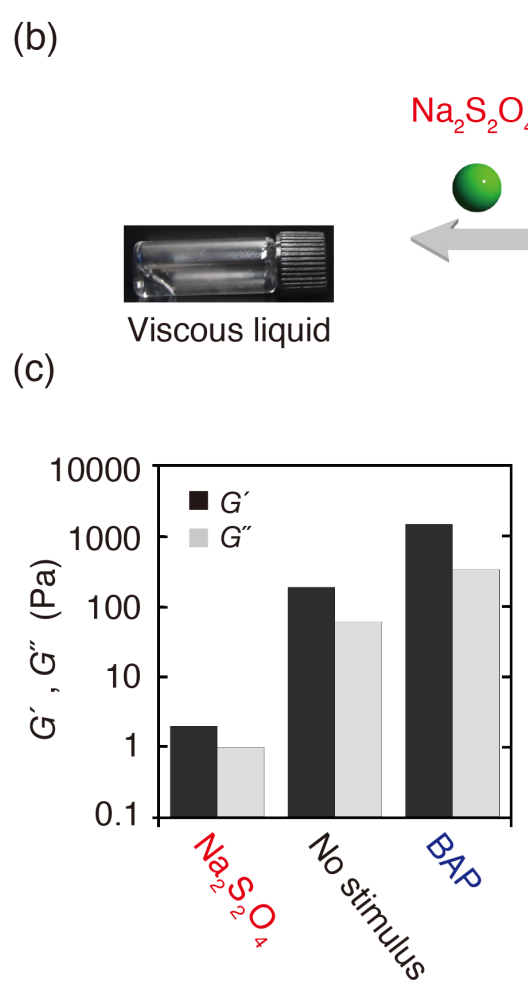

\section{NPmoc-F(4-F)F \\ Phos-cycC 6}

Mechanical strength

Protein release rate

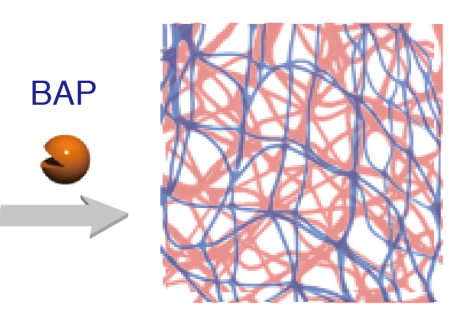

NPmoc-F(4-F)F

Phos / HO-cycC 6

Higher

Lower (d)

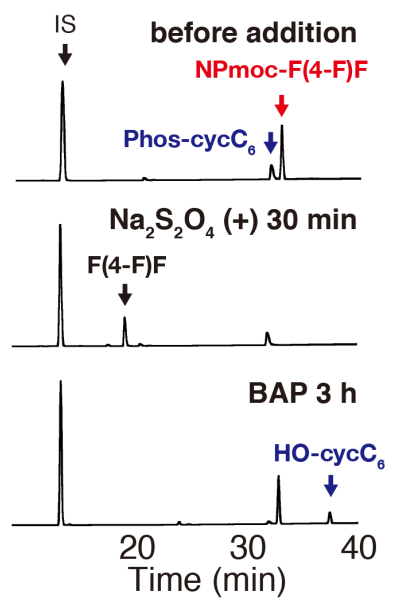

(e)

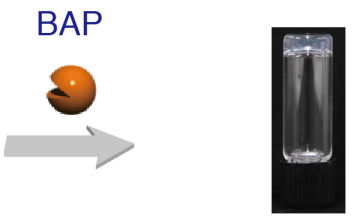

Harder gel

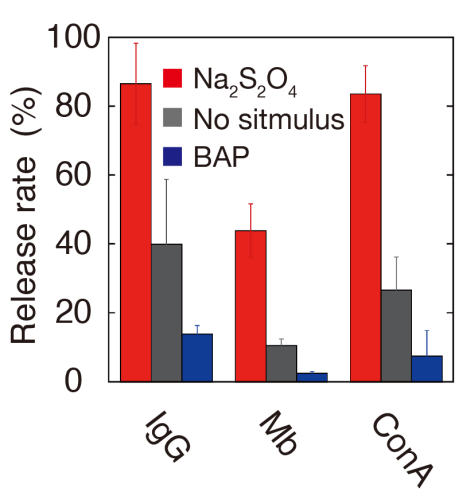


1 Figure 5. Stimuli-responsiveness of the self-sorting double network (SDN) hydrogel. (a)

2 Schematic representation of orthogonal hybridization of the expected responses towards

$3 \mathrm{Na}_{2} \mathrm{~S}_{2} \mathrm{O}_{4}$ and BAP into the SDN hydrogel. The blue, yellow, and red colours indicate

4 NPmoc-F(4-F)F, Phos-cycC 6 , and Phos / HO-cycC 6 networks, respectively. (b) Optical

5 photos of the SDN hydrogels (middle) and the viscous liquid and gel after addition of

$6 \quad \mathrm{Na}_{2} \mathrm{~S}_{2} \mathrm{O}_{4}$ (left) and BAP (right), respectively. (c) Rheological properties of the SDN hydrogel

7 at a frequency of $10 \mathrm{rad} / \mathrm{s}$ before and after addition of stimuli (left: $\mathrm{Na}_{2} \mathrm{~S}_{2} \mathrm{O}_{4}$ (30 min), middle:

8 no stimulus, right: BAP $(3 \mathrm{~h})$ ). The control experiments, the frequency dependences of the

9 gel samples and the reason of larger $G^{\prime}$ than $G^{\prime \prime}$ of the viscous liquid obtained after addition

10 of $\mathrm{Na}_{2} \mathrm{~S}_{2} \mathrm{O}_{4}$ to the SDN hydrogel are shown and discussed in Supplementary Figures 21-25.

11 (d) HPLC charts of the SDN hydrogels (upper: before addition, middle: $\mathrm{Na}_{2} \mathrm{~S}_{2} \mathrm{O}_{4}(30 \mathrm{~min})$,

12 lower: BAP $(3 \mathrm{~h})$ ). (e) Protein release rate from the SDN hydrogels after addition of stimuli

13 (left: IgG, middle: $\mathrm{Mb}$, right: ConA, red: $\mathrm{Na}_{2} \mathrm{~S}_{2} \mathrm{O}_{4}$ (30 min), gray: no stimulus, blue: BAP (3

14 h)). The data represent mean \pm s.d.m $(n=3)$. Conditions: $[\mathbf{N P m o c F}(4-\mathbf{F}) \mathbf{F}]=0.40 \mathrm{wt} \%(7.9$

$15 \mathrm{mM}),[$ Phos-cycC 6$]=0.15 \mathrm{wt} \%(2.4 \mathrm{mM}),\left[\mathrm{Na}_{2} \mathrm{~S}_{2} \mathrm{O}_{4}\right]=79 \mathrm{mM}(10$ eq. for NPmoc-F(4-F)F $)$,

$16[\mathrm{BAP}]=0.05 \mathrm{U} / \mu \mathrm{L}$ for $(\mathrm{b}, \mathrm{c}, \mathrm{d})$ and $0.005 \mathrm{U} / \mu \mathrm{L}$ for $(\mathrm{e})$ in $100 \mathrm{mM}$ HEPES buffer $(\mathrm{pH} \mathrm{8.0)}$.

$17 V_{\text {gel }} / V_{\text {stimulus }}=10: 1,25^{\circ} \mathrm{C}$. In Figure (e), the protein stock solutions $(0.5 \mathrm{mg} / \mathrm{mL}$ IgG, 1.0

$18 \mathrm{mg} / \mathrm{mL}$ ConA, $0.5 \mathrm{mg} / \mathrm{mL}$ myoglobin, $10 \mu \mathrm{L})$ were added to SDN hydrogels $(200 \mu \mathrm{L})$. 
(a)

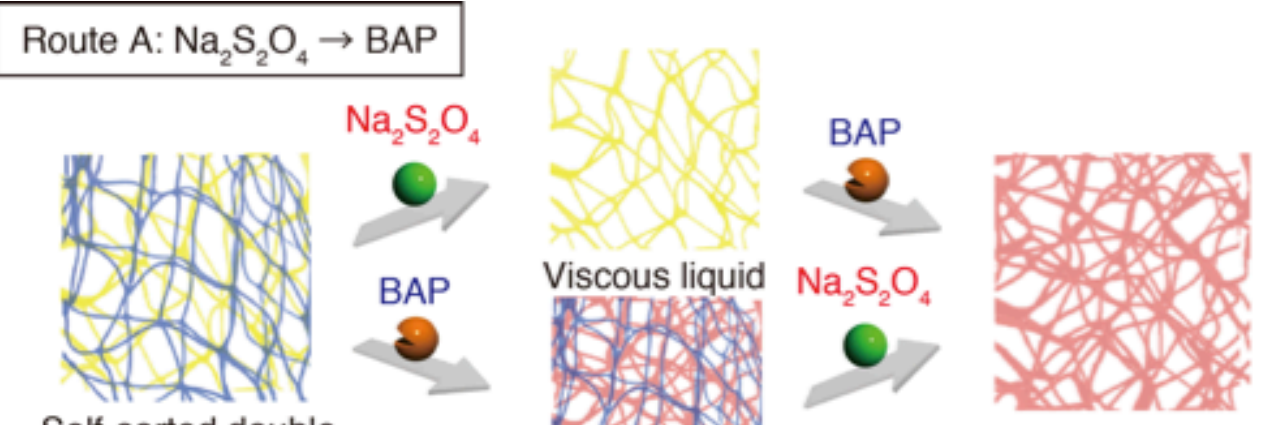

Self-sorted double network hydrogel

Route B: BAP $\rightarrow \mathrm{Na}_{2} \mathrm{~S}_{2} \mathrm{O}_{4}$

(b) Route A
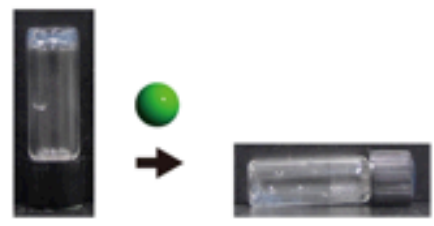

Gel Sol

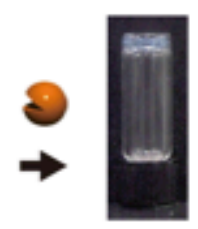

Gel

(d) Route A

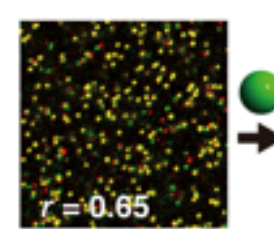

OFF

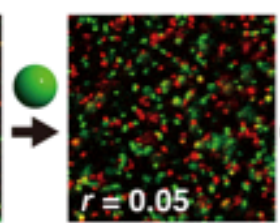

ON

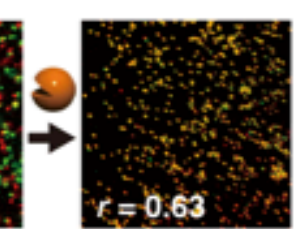

OFF (c) Route B

(f)
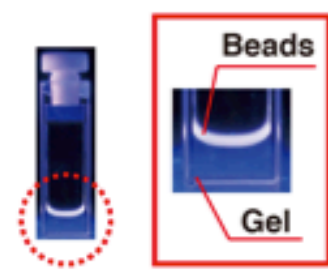

Self-sorting double network (SDN) hydrogel

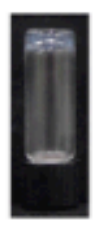

Gel

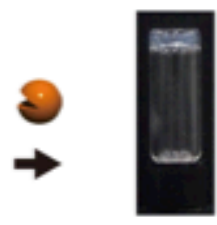

Gel

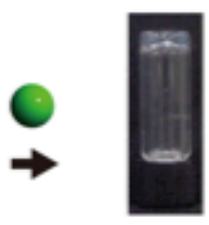

Gel

(e) Route B

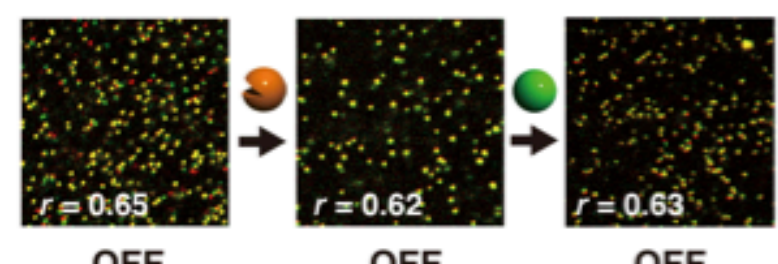

OFF

OFF

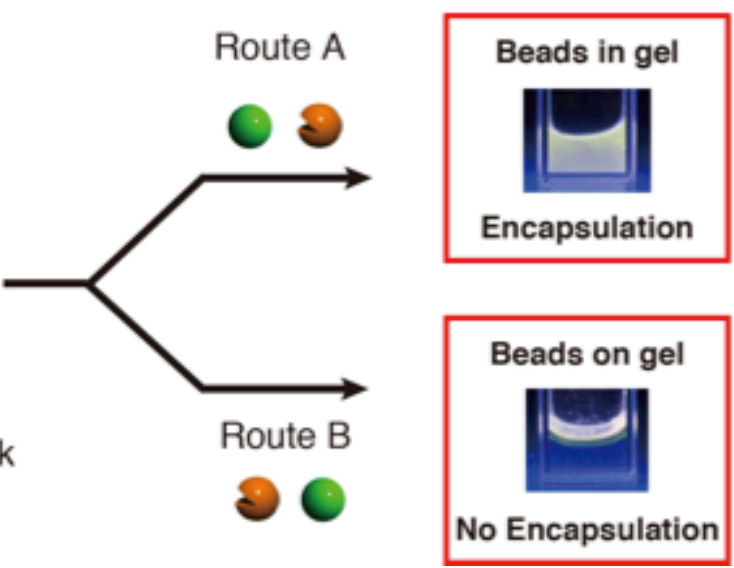


1 Figure 6. Recognition of the stimulus order by the SDN hydrogel. (a) Schematic 2 representation of recognition of the stimulus order by the SDN hydrogels. The upper line 3 shows the expected changes of the SDN hydrogel after addition of $\mathrm{Na}_{2} \mathrm{~S}_{2} \mathrm{O}_{4}$ and then BAP 4 (route A). The lower line shows the reverse addition order (route B). The blue, yellow, and 5 red colours indicate NPmoc-F(4-F)F, Phos-cycC 6 , and Phos / HO-cycC 6 networks, 6 respectively. (b, c) Optical photos of the SDN hydrogels after addition of stimuli (b: route A, 7 c: route B). (d, e) Brownian motion of beads (diameter: $300 \mathrm{~nm}$ ) in the SDN hydrogels before 8 and after addition of stimuli (d: route A, e: route B). The images are merged images at 0 and $91 \mathrm{~min}$ after starting CLSM observations. The original CLSM images at 0 and $1 \mathrm{~min}$ are 10 shown in Supplementary Figures 35 and 36. The numbers in the images represent Pearson's 11 correlation coefficient $(r)$. [Fluorescence nanobeads $(300 \mathrm{~nm})]=0.05 \mathrm{mg} / \mathrm{mL}$. (f) Optical 12 photos of bead encapsulation experiments (upper: route A, lower: route B) under UV light $13(254 \mathrm{~nm})$. The left photo in a red box shows the magnified image of the hydrogel and 14 fluorescence beads before addition of stimuli. The right photos indicate the hydrogels after addition of stimuli (upper: route A, lower: route B). In the case of route A, fluorescence beads were encapsulated in the resultant hydrogels. On the other hand, in route B, the beads were not encapsulated in the hydrogels. Conditions: $[\mathbf{N P m o c F}(4-\mathbf{F}) \mathbf{F}]=0.40 \mathrm{wt} \%(7.9 \mathrm{mM})$, 18 [Phos-cycC 6$]=0.15 \mathrm{wt} \%(2.4 \mathrm{mM}),\left[\mathrm{Na}_{2} \mathrm{~S}_{2} \mathrm{O}_{4}\right]=79 \mathrm{mM}(10$ eq. for NPmoc-F(4-F)F $)$, $[\mathrm{BAP}]=0.05 \mathrm{U} / \mu \mathrm{L}$ in $100 \mathrm{mM}$ HEPES buffer $(\mathrm{pH} 8.0) . V_{\text {gel }} / V_{\text {stimulus }}=10: 1,25^{\circ} \mathrm{C}$. 


\section{Methods}

2 The materials, instruments, experimental methods and syntheses of the compounds are

3 described in supplementary material. The conditions (concentration, $\mathrm{pH}$ and temperature) of

4 the experiments are provided in the figure captions. The authors declare that the data that

5 support the findings of this study are available within this paper and its supplementary

6 information files.

7

\section{$8 \quad$ Additional information}

9 Supplementary information is available in the online version of the paper. Reprints and

10 permission information is available online at www.nature.com/reprints. Correspondence and

11 requests for materials should be addressed to I. H. 